\title{
1 A Novel Mistranslating tRNA Model in Drosophila melanogaster has Diverse,
}

\section{Sexually Dimorphic Effects}

4 Joshua R. Isaacson ${ }^{*}$, Matthew D. Berg ${ }^{\dagger,+}$, Jessica Jagiello*, Judit Villén ${ }^{\dagger}$, Christopher J. Brandl ${ }^{\dagger}$

6 *Department of Biology and ${ }^{\dagger}$ Department of Biochemistry, The University of Western Ontario,

$7 \quad$ N6A 5B7, London, Canada

8 Department of Genome Sciences, University of Washington, Seattle, Washington, 98195 
10 Running title: Mistranslation impairs development

11 Key words: tRNA, mistranslation, Drosophila melanogaster, development, locomotion,

12 proteostasis, deformity, sex-specific

13 Corresponding author: Joshua Isaacson, 1151 Richmond Street, Biological and Geological

14 Sciences Building Room 2082, London, Ontario, N6A 5B7, Canada, email: jisaacso@ uwo.ca

15 phone: $519-661-2111 \times 85596$ 


\section{ABSTRACT}

Transfer RNAs (tRNAs) are the adaptor molecules required for reading of the genetic

18 code and the accurate production of proteins. tRNA variants can lead to genome-wide

19 mistranslation, the misincorporation of amino acids not specified by the standard genetic code

20 into nascent proteins. While genome sequencing has identified putative mistranslating tRNA

21 variants in human populations, little is known regarding how mistranslation affects multicellular

22 organisms. Here, we create a Drosophila melanogaster model for mistranslation by integrating a

23 serine tRNA variant that mistranslates serine for proline (tRNA ${ }_{\text {UGG, G26A }}^{\mathrm{Ser}}$ into the fly genome.

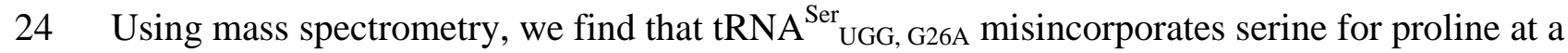

25 frequency of $\sim 0.6 \%$ per codon. We find that mistranslation extends development time and

26 decreases the number of flies that reach adulthood. Adult flies containing tRNA ${ }_{\text {UGG, G26A }}^{\text {Ser }}$

27 present with more morphological deformities and worse climbing performance than flies

28 expressing only wild type tRNA. Female flies with the serine tRNA variant have more

29 deformities and experience a faster decline in climbing performance than males, suggesting sex-

30 specific effects. This model will enable studies into the synergistic effects of mistranslating

31 tRNA variants and disease-causing alleles.

\section{INTRODUCTION}

Mistranslation occurs when an amino acid that differs from what is specified by the

34 standard genetic code is incorporated into nascent proteins. Mistranslation disrupts proteostasis

35 and impairs cell function and growth (Nangle et al. 2006; Paredes et al. 2012; Reverendo et al.

36 2014; Liu et al. 2014; Shcherbakov et al. 2019), yet naturally occurs in all cells at rates of $10^{-2}$ to

$3710^{-5}$ per codon, depending on the codon (reviewed in Joshi et al. 2019). Protein quality control

38 pathways allow cells to tolerate mistranslation at frequencies approaching $10 \%$ in 
Saccharomyces cerevisiae and Escherichia coli (Ruan et al. 2008; Berg et al. 2019b).

40 Mistranslating tRNA variants are also tolerated because of the buffering provided by multiple

41 copies of the genes encoding tRNAs in most organisms (e.g. 610 total tRNA genes in humans

42 and 295 total tRNA genes in Drosophila melanogaster, Chan and Lowe 2016). Mistranslation

43 can be an adaptive response. For example, high levels of mistranslation increase survival of $E$.

44 coli exposed to DNA damage (Samhita et al. 2020) and misincorporation of methionine protects

45 mammalian, yeast, and bacterial cells against reactive oxygen species (Netzer et al. 2009;

46 Wiltrout et al. 2012). recognition increase mistranslation frequency. The fidelity of aminoacylation depends on aminoacyl-tRNA synthetases (aaRSs) correctly recognizing their cognate tRNAs through nucleotides, base pairs, and structural elements within the tRNA called identity elements. The

51 anticodon is the main identity element for all tRNAs except tRNA ${ }^{\text {Ser }}$, RNA $^{\text {Ala }}$ and tRNA ${ }^{\text {Leu }}$

52 (Mcclain and Foss 1988; Hou and Schimmel 1988; Normanly et al. 1992; Asahara et al. 1993;

53 Achsel and Gross 1993; Breitschopf et al. 1995; Himeno et al. 1997; Giegé et al. 1998).

54 Changing the anticodon of tRNA ${ }^{\text {Ser }}$ does not affect aminoacylation but changes codon

55 recognition (Garza et al. 1990; Geslain et al. 2010; Reverendo et al. 2014; Zimmerman et al.

56 2018; Berg et al. 2019b).

Mutations in tRNAs that cause mistranslation arise spontaneously and were identified

58 initially in E. coli as suppressors of nonsense and missense mutations (see for examples; Stadler

59 and Yanofsky 1959; Gorini and Beckwith 1966; Goodman et al. 1968). Subsequently,

60 mistranslating tRNAs have been identified through their suppression of deleterious phenotypes

61 in fungi, nematodes, plants, and mammalian cells (e.g Goodman et al. 1977; Wills et al. 1983; 
62 Chiu and Morris 1997; El Meziane et al. 1998; Murakami et al. 2005). While no spontaneous

63 tRNA variants have been detected through suppression screens in Drosophila, Laski et al. (1989)

64 and Garza et al. (1990) have engineered amber suppressing tRNA ${ }^{\text {Tyr }}$ and tRNA ${ }^{\text {Leu }}$ variants,

65 respectively, that show a low level of amber stop codon suppression activity when integrated into

66 the Drosophila melanogaster genome. In both cases sterility was noted.

In humans, mistranslation due to tRNA variants can cause disease (Goto et al. 1990;

69 reviewed in Kapur and Ackerman 2018 and Lant et al. 2019). Yet tRNA variants are relatively

70 common in humans, with $~ 66$ tRNA variants per individual, some of which are predicted to

71 decrease translational fidelity (Berg et al. 2019a). Despite the prevalence of potential

72 mistranslating tRNAs and the potential links between mistranslation and disease, the impact of

73 mistranslating cytoplasmic tRNAs in multicellular organisms is not well described. In this study,

74 we develop a transgenic model of mistranslation in D. melanogaster by genomically integrating

75 a serine tRNA variant that mistranslates serine at proline codons. We hypothesize that loss of

76 proteostasis caused by the mistranslating tRNA will impact development, fitness, and behaviour.

77 Serine for proline substitutions were detected by mass spectrometry in pupae expressing the

78 mistranslating tRNA variant. Development time of flies containing the mistranslating tRNA was

79 extended and fewer flies reached adulthood compared to wild type flies. The tRNA variant

80 increased the prevalence of morphological deformities in adult flies, with females being more

81 severely affected than males. Mistranslation also impaired climbing performance. These results

82 demonstrate that D. melanogaster provides a model for the impact and genetic interactions of

83 mistranslating tRNAs in a multicellular organism and their sexually dimorphic effects. 


\section{METHODS}

85

86

87

88

89

90

91

92

93

94

95

96

97

98

99

100

101

102

103

104

105

Fly husbandry and stocks

\section{Creating transgenic stocks}

All fly stocks were obtained from the Bloomington Drosophila Stock Centre and maintained on standard Bloomington recipe food medium (BDSC; Bloomington, Indiana) under a 14:10 light:dark cycle at $24^{\circ} \mathrm{C}$ and $70 \%$ relative humidity.

The gene encoding wild type tRNA ${ }_{\text {UGA }}^{\text {Ser }}$ (FlyBase ID: FBgn0050201) was amplified from D. melanogaster genomic DNA using primers VK3400/VK3401 (primers are listed in Table S1) and cloned into pCDF4, which was a kind gift from Dr. Simon Bullock (Port et al. 2014) as a $B g l \mathrm{II} / \mathrm{XbaI}$ fragment to create $\mathrm{pCB} 4222$. The gene encoding a variant tRNA with a

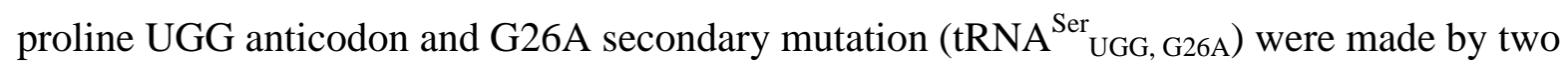
step mutagenic PCR with primers VK3400/VK3889 and VK3401/VK3890 in the first round and pCB4222 as a template. Products from the first round were amplified with outside primers VK3400/VK3401 and cloned as a BglII/XbaI fragment into pCDF4 to give pCB4250. Full

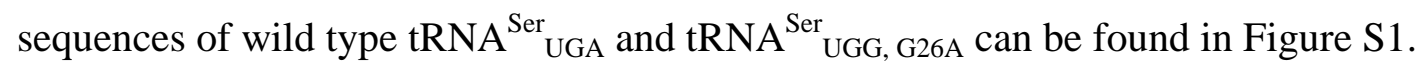

To create flies containing mistranslating tRNAs, a stock expressing phiC31 (ФC31) integrase in the germ line and containing an attP site in the left arm of the second chromosome was used (stock \# 25709: $y^{1} v^{l} P\{$ nos-phiC31 int.NLS\}X; P\{CaryP $\}$ attP40). Plasmids were injected into D. melanogaster embryos using the protocol described in Isaacson (2018).

Transgenic flies were identified by their wild type eye colour and balanced using stock \# 3703 $\left(w^{1118} / \mathrm{Dp}(1 ; Y) y^{+} ;\right.$CyO/nub $b^{1} b^{1}$ sna Sco $l t^{1}$ stw $^{3} ;$ MKRS/TM6B, Tb $\left.{ }^{1}\right)$ and \#76359 $\left(w^{1118} ; w^{S p-}\right.$ $\left.{ }^{1} / C y O, P\left\{w^{+m C}=2 x T b^{1}-R F P\right\} C y O ; M K R S / T M 6 B, T b^{1}\right)$ to create final stocks of the following 
106

107

108

109

genotype: $w^{1118} ; P\{C a r y P\} a t t P 40\left[v^{+}=t R N A\right] / C y O, P\left\{w^{+} m C=2 x T b^{1}-R F P\right\} C y O ; M K R S / T M 6 B$, $T b^{l}$. After producing offspring, DNA was extracted from both parents of the final cross and PCR amplified using the primer set M13R and VK3400. PCR products were sequenced to confirm tRNA identity.

Complementation in Saccharomyces cerevisiae

The $B g l \mathrm{II} / X b a \mathrm{I}$ fragment of pCB422 encoding Drosophila $\mathrm{tRNA}_{\text {UGG, G26A }}^{\mathrm{Ser}}$ was cloned into the BamHI/XbaI sites of the yeast-E. coli shuttle plasmid YEPlac181 (Gietz and Sugino 1988; LEU2, 2 micron; CB4877). CB4877 and YEPlac181 were transformed into the yeast strain

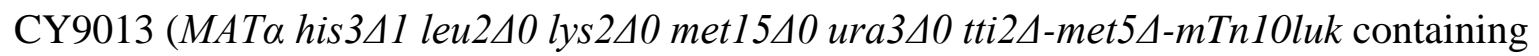
pRS313 (Sikorski and Hieter 1989) expressing tti2-L187P (Berg et al. 2017) selecting for growth on minimal plates lacking leucine and histidine. Transformants were streaked onto yeast-peptone (YP) plates containing $2 \%$ glucose and $5 \%$ ethanol and grown at $30^{\circ} \mathrm{C}$ for 4 days.

\section{Mass spectrometry}

Six replicates of twenty pupae were collected from each genotype and lysed in $8 \mathrm{M}$ urea, $50 \mathrm{mM}$ Tris, $75 \mathrm{mM} \mathrm{NaCl}, \mathrm{pH} 8.2$ by grinding with a pestle and with glass beads at $4^{\circ} \mathrm{C}$. Protein was reduced with $5 \mathrm{mM}$ dithiothreitol for 30 minutes at $55^{\circ} \mathrm{C}$ and alkylated with $15 \mathrm{mM}$ iodoacetamine for 30 minutes at room temperature. Robotic purification and digestion of proteins into peptides were performed on the KingFisher ${ }^{\mathrm{TM}}$ Flex using LysC and the R2-P1 method as described in Leutert et al. (2019). Peptides were separated by reverse-phase chromatography and online analyzed on a hybrid quadrupole orbitrap mass spectrometer (Orbitrap Exploris 480; Thermo Fisher Scientific) operated in data-dependent acquisition mode as described in Berg et al. (2021). 
MS/MS spectra were searched against the D. melanogaster protein sequence database

129 (downloaded from Uniprot in 2016) using Comet (release 2015.01; Eng et al. 2013). The

130 precursor mass tolerance was set to $50 \mathrm{ppm}$. Constant modification of cysteine

131 carbamidomethylation (57.0215 Da) and variable modification of methionine oxidation (15.9949

$132 \mathrm{Da})$ and proline to serine (-10.0207 Da) were used for all searches. A maximum of two of each

133 variable modification were allowed per peptide. Search results were filtered to a $1 \%$ false

134 discovery rate at the peptide spectrum match level using Percolator (Käll et al. 2007). The

135 mistranslation frequency was calculated using the unique mistranslated peptides for which the

136 non-mistranslated sibling peptide was also observed. The frequency is defined as the counts of

137 mistranslated peptides, where serine was inserted for proline, divided by the counts of all

138 peptides containing proline, respectively, and expressed as a percentage.

140 Virgin, heterozygous flies were collected within $\sim 8$ hours of eclosion and scored for

141 deformities in adult legs (limbs gnarled or missing segments), wings (blistered, absent, fluid-

142 filled, or abnormal size), or abdomen (fused or incomplete tergites). Flies collected before wing

143 expansion were excluded. Sex and type of deformity was recorded. Flies that had multiple

144 deformities had each recorded. 433 tRNA ${ }_{\text {UGA }}^{\text {Ser }}$ flies (227 males and 216 females) and 656

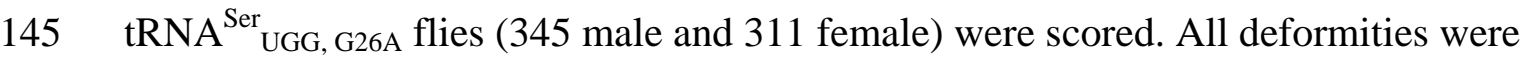

146 photographed through the lens of a stereomicroscope using a Samsung Galaxy S8 camera.

147 Developmental assays

148 Approximately 250 flies of each genotype were placed into fly cages and allowed to lay 149 eggs for one hour. Three replicates of 30 eggs from each plate were collected and checked every 
15012 hours to record progress through each of the following developmental stages: egg hatching

151 into larva, larva pupating into pupa, and adult eclosing from pupa. Sex, zygosity, and deformities

152 (as described above) were recorded.

153 Climbing assays

154 Virgin adult flies were collected, sorted by sex, and scored for deformities. Deformed

155 flies or flies homozygous for the transgenic tRNA were discarded. Equal numbers of flies were

156 collected from each genotype during each collection period. Sixty flies in 11 vials from each

157 genotype were collected and transferred to new food the day before testing. The number of flies

158 that climbed to a $5 \mathrm{~cm}$ line in 10 seconds was recorded, and flies were retested every three days

159 until the flies were 51 days old. Each vial was tested three times.

160 Statistical analyses

161 All statistical analyses were performed using R Studio 1.2.5001. Frequency of proline-to-

162 serine misincorporation between $\mathrm{tRNA}_{\mathrm{UGA}}^{\mathrm{Ser}}$ and $\mathrm{tRNA}{ }_{\mathrm{UGG}}^{\mathrm{Ser}}$ G26A was compared using a $t$-test.

163 Developmental time data were compared using Wilcoxon rank-sum tests. Fisher's exact tests

164 were used to compare survival between developmental stages and proportion of deformities. Chi-

165 square tests were used to compare prevalence of each type of deformity using a post hoc analysis

166 outlined in Shan and Gerstenberger (2017). A generalized linear model was constructed from the

167 climbing assay data and performance was compared using F-tests.

\section{RESULTS}

$169 A t R N A^{\text {Ser }}$ variant induces mistranslation in Drosophila melanogaster 
To characterize mistranslation in a multicellular organism, we integrated genes encoding

171 wild type tRNA ${ }_{\text {UGA }}^{\text {Ser }}$ as a control and a tRNA ${ }^{\text {Ser }}$ variant that mistranslates serine for proline

172 (Figure 1A) into the left arm of the second chromosome of the D. melanogaster genome. The

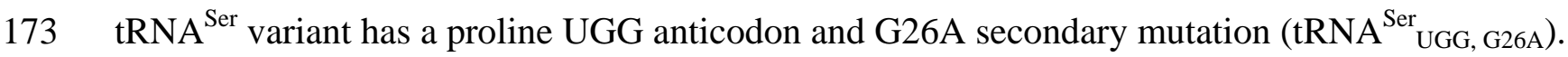

174 The alleles were balanced over a homolog that has serial inversions, preventing recombinant

175 offspring and transgene loss. tRNA insertions were validated with PCR using primers specific to

176 the inserted plasmid and confirmed by sequencing. The secondary G26A mutation was included

177 in the mistranslating tRNA to dampen tRNA function as we have previously found a tRNA ${ }^{\text {Ser }}$

178 variant with a proline anticodon causes lethal levels of mistranslation when expressed in yeast

179 (Berg et al. 2017).

Adults homozygous for $\mathrm{tRNA}^{\mathrm{Ser}} \mathrm{UGA}_{\mathrm{A}}$ or tRNA ${ }_{\text {UGG, G26A }}^{\mathrm{Ser}}$ can be produced. However, we

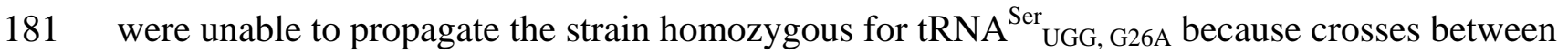

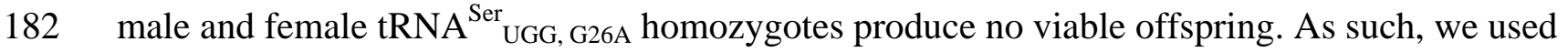

183 heterozygous flies for our experiments with adults. Studying heterozygous flies may be more

184 biologically relevant as mistranslating tRNAs present in populations are likely to arise as single

185 alleles. We determined zygosity by balancing the tRNAs over a $C y O$ homolog containing Tubby-

186 linked RFP and miniwhite (Pina and Pignoni 2012). Heterozygous larvae and pupae are

187 identified by the presence of RFP and heterozygous adults by their curly wings and non-white

188 eyes.

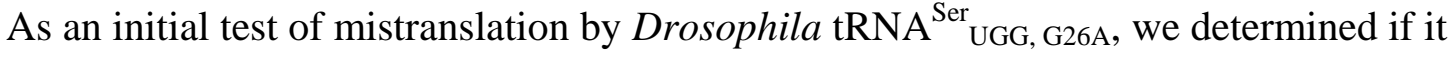

190 would rescue the growth of a S. cerevisiae strain containing tti2-L187 (CY4013). The tti2-L187

191 allele contains a missense mutation converting a CUA codon for leucine to CCA for proline and

192 results in the slow growth of yeast in stress conditions including in medium containing 5\% 
193 ethanol (Hoffman et al. 2017). Mistranslation of proline to serine rescues the growth of yeast

194 cells in ethanol medium (Berg et al. 2017). The gene encoding Drosophila tRNA ${ }_{\text {UGG, G26A Was }}^{\text {Ser }}$

195 transformed into yeast strain CY4013 that contains tti2-L187 as the sole copy of TTI2. Cells were

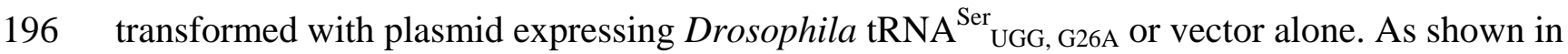

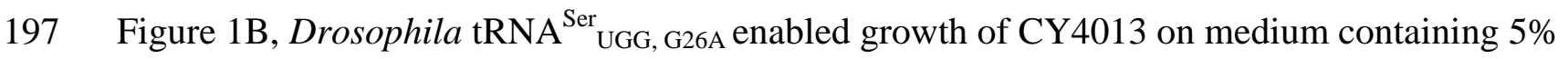

198 ethanol indicative of mistranslation by Drosophila tRNA ${ }_{\text {UGG, G26A. }}^{\text {Ser }}$

199 We then analyzed the proteome of $D$. melanogaster pupae by mass spectrometry to

200 determine the mistranslation frequency (Figure 1C; Supplemental File S2). Pupae were used

201 because of the extensive cellular remodelling and corresponding rapid changes in protein

202 synthesis that occur during this stage (Mitchell et al. 1977; Mitchell and Petersen 1981), and the

203 potential of mistranslation during this stage to influence adult traits such as anatomy or neuronal

204 function. The frequency of proline to serine mistranslation calculated as the ratio of peptides

205 containing the mistranslated serine residue to peptides containing the cognate proline residue was

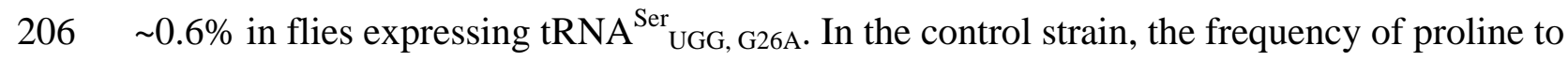

207 serine substitutions was $0.1 \%$. 


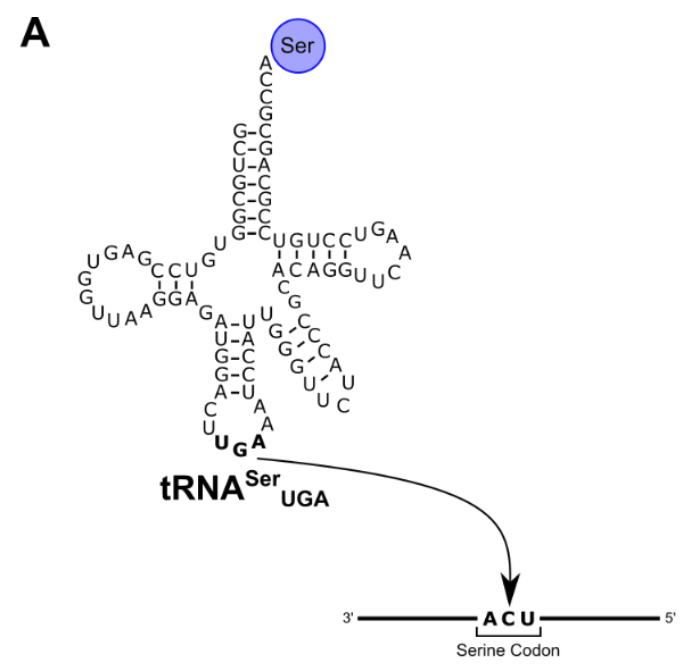

B

\section{YP with 5\% Ethanol}
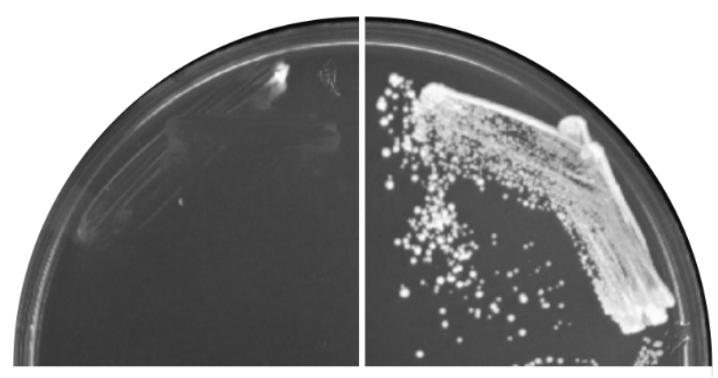

208

209

210

211

212

213 CCA codons and inserts serine at proline codons. Red bases indicate mutation compared to the

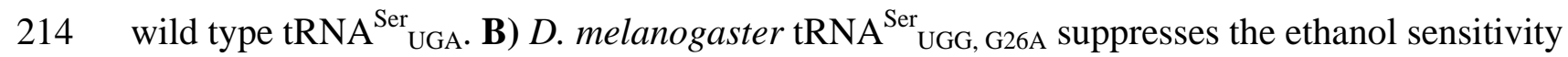

215 caused by tti2-L187P in S. cerevisiae. Plasmid encoding the vector alone (left) or the gene

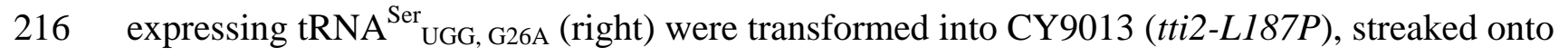

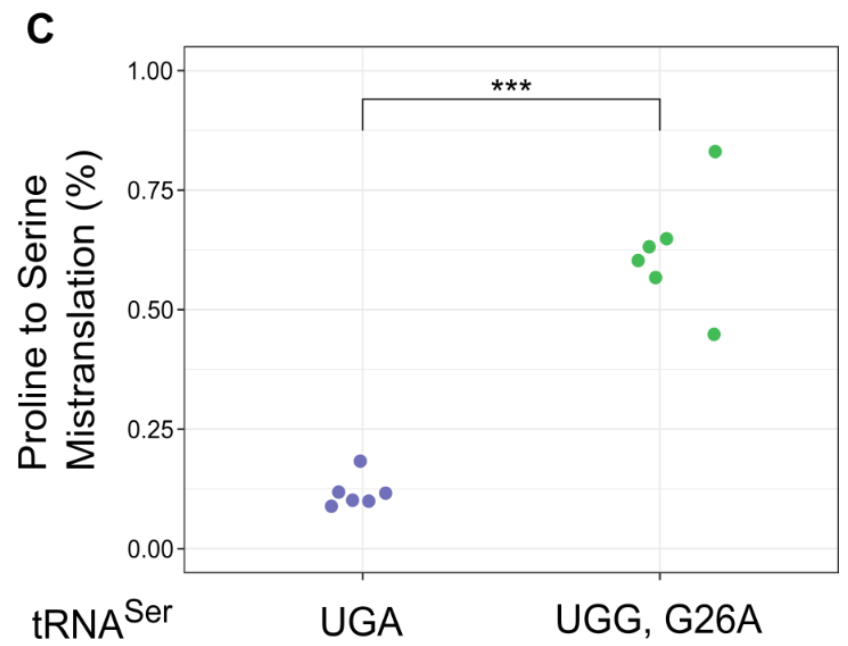

Figure 1. $t R N A_{U G G, G 26 A}^{\operatorname{Ser}}$ induces mistranslation in D. melanogaster. A) Wild type serine tRNAs base pair with serine codons during translation and incorporate serine into the growing polypeptide. Serine tRNA variants with anticodon mutations can recognize non-serine codons and misincorporate serine during translation. $\mathrm{tRNA}^{\mathrm{Ser}}{ }_{\mathrm{UGG}}, \mathrm{G} 26 \mathrm{~A}$ competes with $\mathrm{tRNA}_{\text {UGG for }}^{\text {Pro }}$ 
217 YP medium containing 5\% ethanol and grown at $30^{\circ}$ for 4 days. C) Frequency of proline-to-

218 serine mistranslation in tRNA ${ }_{\text {UGA }}^{\text {Ser }}$ and $\mathrm{tRNA}_{\mathrm{UGG}}^{\mathrm{Ser}}$,26A pupae $(\mathrm{n}=6$ replicates of 20 pupae

219 each). Genotypes were compared using a $t$-test. “***” $\mathrm{p}<0.001$.

221 Mistranslation adversely affects D. melanogaster development

To determine if tRNA ${ }_{\text {UGG, G26A }}^{\text {Ser }}$ affects fly development, we collected 90 one-hour old

223 embryos and monitored how many individuals survived through each developmental stage and

224 time between each stage: egg laying to embryos hatching into larvae, hatching to pupation, and

225 pupation to eclosion of adults. Since the RFP marker used to determine tRNA zygosity is not

226 expressed at early embryonic stages, both homozygotes and heterozygotes were pooled in this

227 assay. Flies were checked every twelve hours and sex, zygosity, and presence of deformities in

228 adults were recorded (Supplemental file S2). No obvious patterns were noted regarding these

229 traits and because too few adults eclosed in this experiment, statistical comparisons were not

230 possible.

Of the 90 embryos collected, significantly more flies with wild type tRNA ${ }_{\text {UGA }}^{\text {Ser }}$ reached

232 larval (41 vs. 27, Fisher's exact test, $\mathrm{p}=0.045)$, pupal (24 vs. 10, p = 0.013), and adult stages (22

233 vs. 9, $\mathrm{p}=0.017$, Figure $2 \mathrm{~A}$ ) than the mistranslating tRNA ${ }_{\mathrm{UGG}}^{\mathrm{Ser}} \mathrm{G} 26 \mathrm{~A}$. Absolute numbers are

234 influenced by die-off in the preceding life-cycle stage, so proportions were also calculated to

235 assess pupal and adult survival. When comparing the proportion of larvae that pupated, 58\% of

236 tRNA ${ }_{\text {UGA }}^{\text {Ser }}$ and $37 \%$ of tRNA ${ }_{\text {UGG, G26A larvae reached pupation, although this difference was }}^{\text {Ser }}$

237 not statistically significant $(\mathrm{p}=0.13)$. The proportion of adults that eclosed from pupae was

238 virtually identical, $91 \%$ for $\mathrm{tRNA}_{\mathrm{UGA}}^{\mathrm{Ser}}$ and $90 \%$ for tRNA ${ }_{\text {UGG, G26A }}^{\text {Ser }}$ (Supp. File S2). These 
239 data indicate that flies are particularly susceptible to lethal effects of mistranslation during

240 embryogenesis but show increased tolerance once they reach pupation. Although there was no

241 significant difference between proportion of $\mathrm{tRNA}{ }_{\mathrm{UGA}}^{\mathrm{Ser}}$ and $\mathrm{tRNA}{ }_{\mathrm{UGG}}^{\mathrm{Ser}} \mathrm{G} 26 \mathrm{~A}$ larvae that

242 pupated, there was a trend of lower survival in tRNA ${ }_{\text {UGG, G26A }}^{\text {Ser }}$ such that larvae seem to display

243 an intermediate sensitivity phenotype between embryos and pupae.

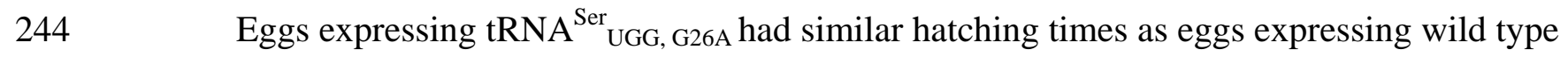

$245 \operatorname{tRNA}^{\text {Ser }}(\mathrm{p}=0.78$, Wilcoxon rank-sum test, Figure $2 \mathrm{~B})$. However, larvae expressing tRNA ${ }_{\text {UGG, }}^{\text {Ser }}$

246 G26A pupated significantly slower than the wild type $(p=0.023$, Figure $2 \mathrm{C})$. This trend continued

247 into adulthood, as the control adult tRNA ${ }_{\text {UGA }}^{\text {Ser }}$ flies eclosed significantly sooner than

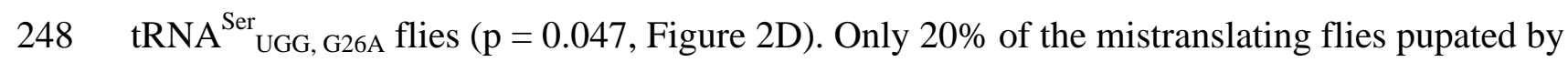

249 the median time for flies with the wild-type tRNA, and only $33 \%$ eclosed by the wild-type tRNA

250 median eclosion time. 
bioRxiv preprint doi: https://doi.org/10.1101/2021.09.17.460863; this version posted September 18, 2021. The copyright holder for this preprint (which was not certified by peer review) is the author/funder, who has granted bioRxiv a license to display the preprint in perpetuity. It is made available under aCC-BY-NC-ND 4.0 International license.

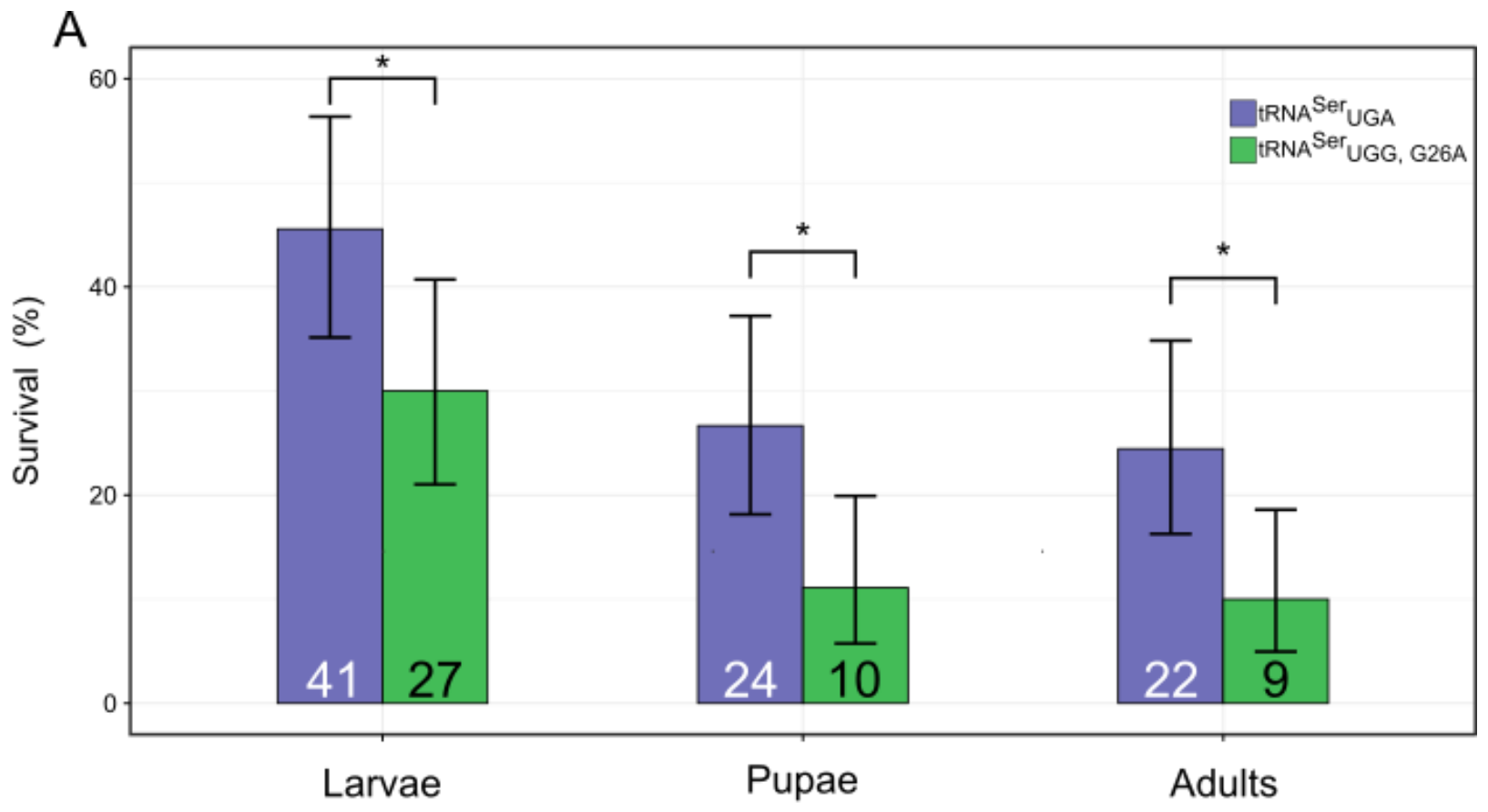

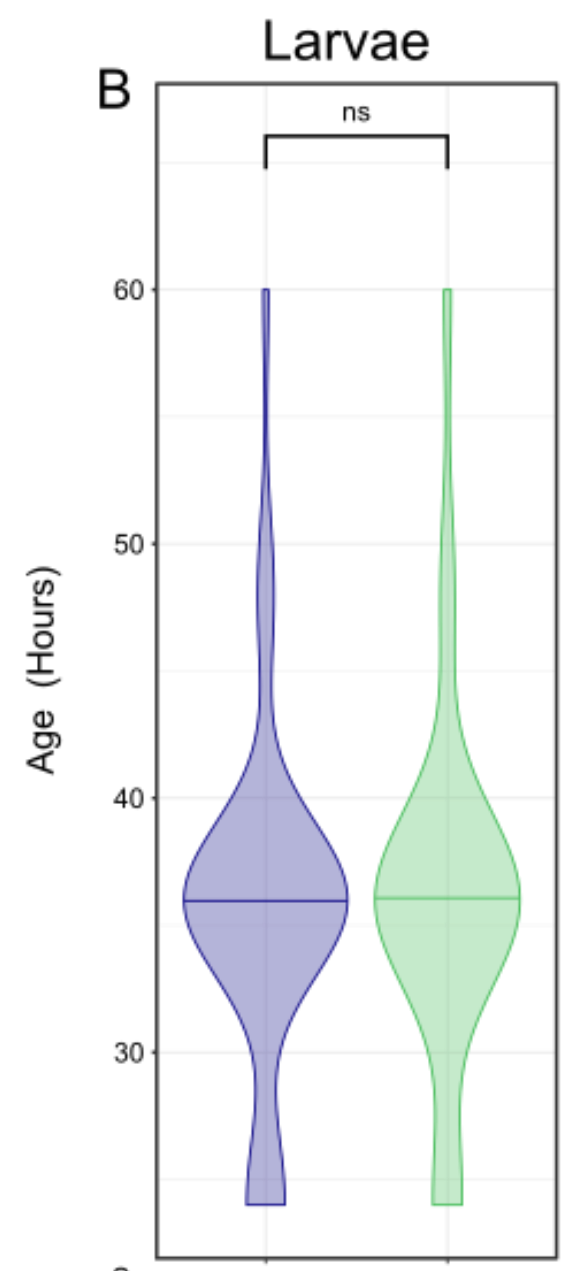

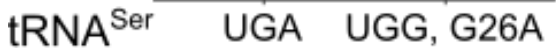

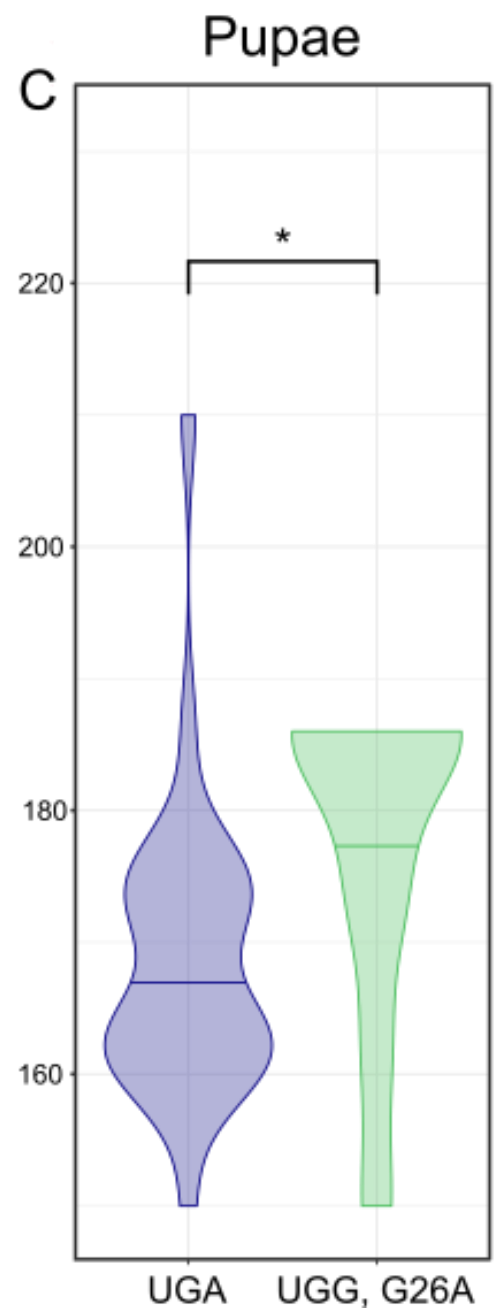

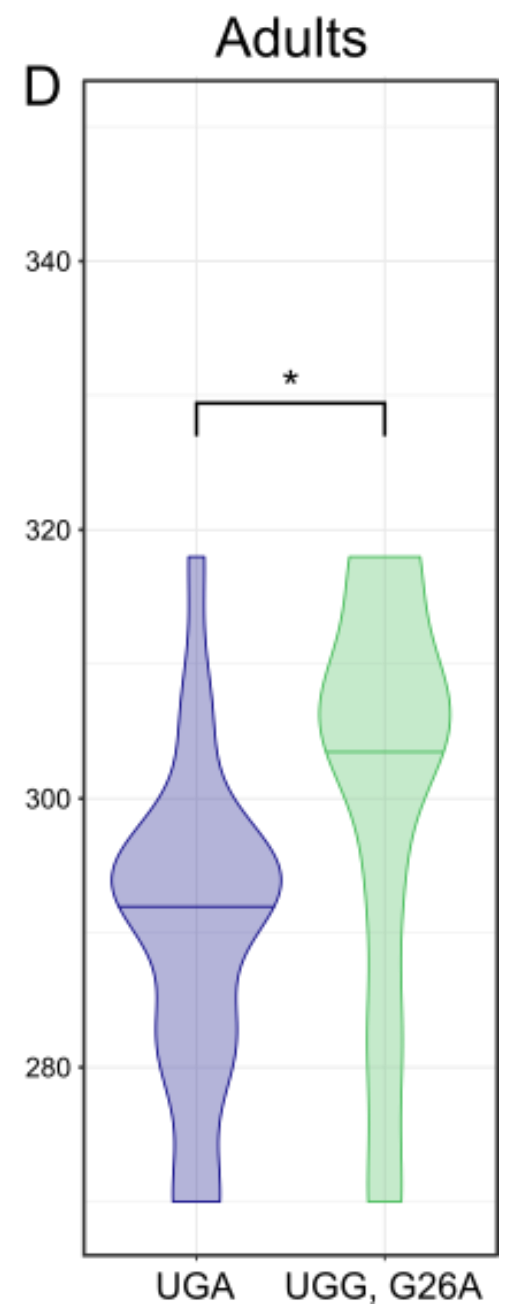


Figure 2. Mistranslation from a tRNA variant impacts development of D. melanogaster. A)

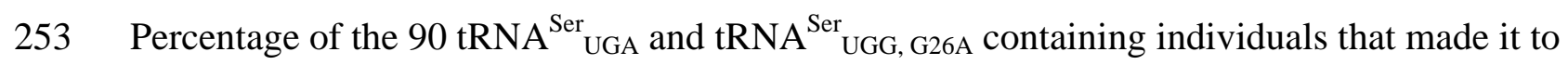

254 larval, pupal, and adult stages. Survival was compared using Fisher's exact test. Error bars

255 represent the $95 \%$ confidence interval of the proportion. Values within bars represent the number

256 of flies that reached that developmental stage. B) Violin plot depicting the distribution of times

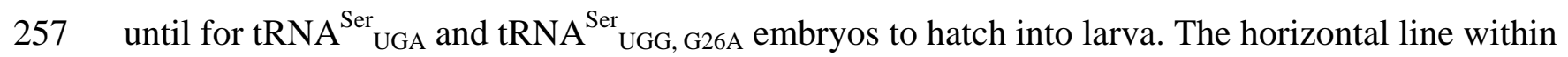

258 the plot represents the median of the distribution. Genotypes were compared using Wilcoxon

259 rank-sum tests. C) Total time until pupation. D) Total time until eclosion. "ns" p $\geq 0.05$, “*” $\mathrm{p}<$

$260 \quad 0.05, “ * * ” p<0.01, “ * * * ” p<0.001$

Mutations in genes vital to proteostasis or translation fidelity cause morphological defects

263 (Rutherford and Lindquist 1998; Cui and DiMario 2007; Reverendo et al. 2014). We observed

264 that flies containing one copy of the exogenous tRNA ${ }_{\text {UGG, G26A }}^{\text {Ser }}$ had deformities including

265 gnarled or blistered legs, notched wings, and misfused tergites (Figure 3A-D). Other

266 abnormalities (e.g. haltere aberrations or rough eyes) were rarely observed, so only the more

267 common leg, wing, and tergite deformities were scored. To determine if the frequency of

268 deformities was greater than the control, we calculated the proportion of flies that eclosed with at

269 least one deformity. These flies were collected separately from the development assay described

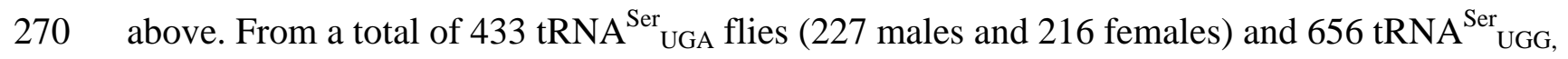

271 G26A flies (345 male and 311 female) we identified significantly more deformities in flies

272 containing tRNA ${ }_{\text {UGG, G26A than }}^{\text {Ser }}$ tRA ${ }_{\text {UGA }}^{\text {Ser }}$ (Fisher's exact test corrected using Holm-

273 Bonferroni's method, $\mathrm{p}<0.001$, Figure $3 \mathrm{E})$. In addition, female flies containing tRNA ${ }_{\text {UGG, G26A }}^{\text {Ser }}$

274 had more deformities than males $(\mathrm{p}<0.001$, Figure 3F). Interestingly, flies containing 


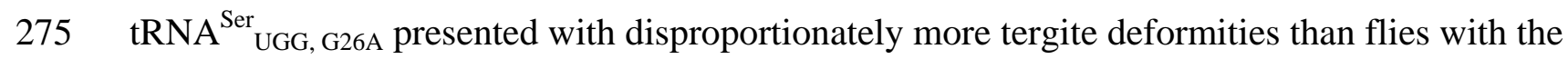

276 wild type tRNA ${ }_{\text {UGA }}^{\text {Ser }}$ (Chi-square test, corrected $\mathrm{p}=0.03$, Supplemental File S3), indicating that

277 this mistranslating tRNA ${ }^{\text {Ser }}$ variant is particularly deleterious to fly abdominal development.

278 These results suggest that mistranslation disrupts fly development and female flies are more

279 sensitive to the presence of mistranslating tRNA variants. 


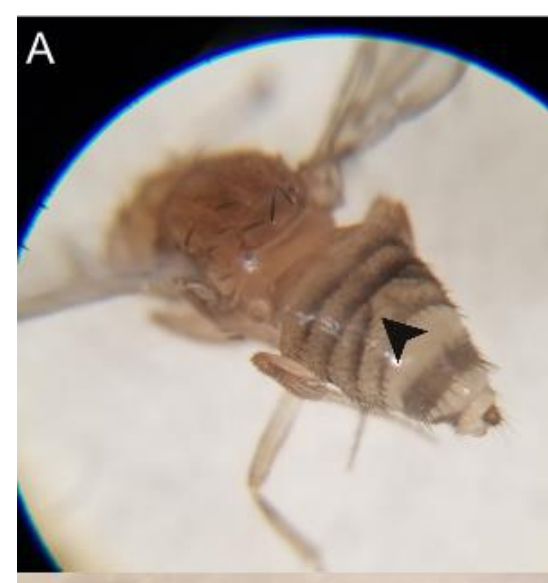

B

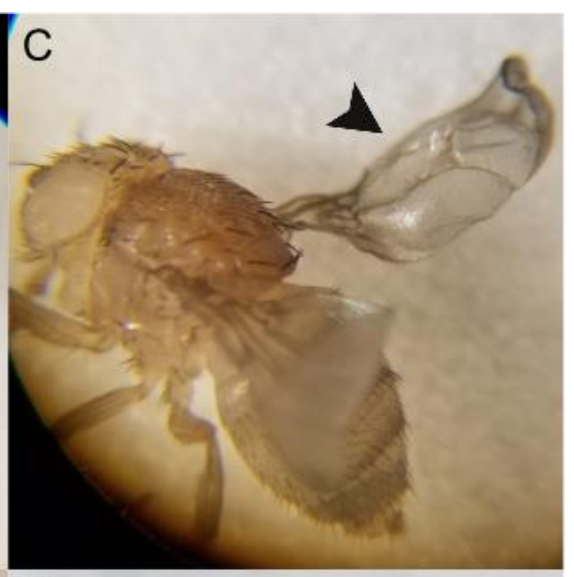

D

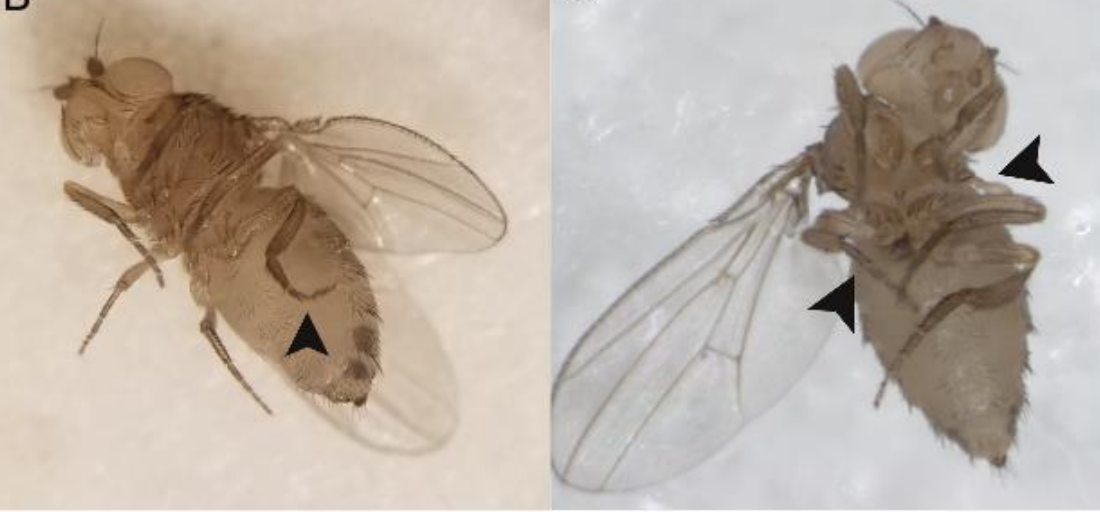

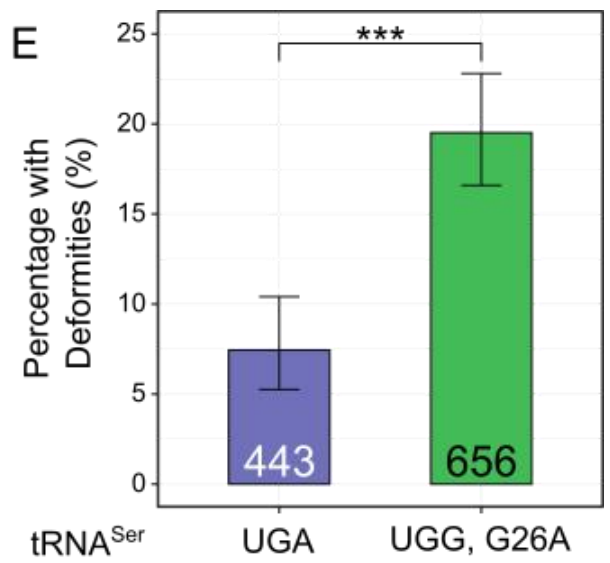

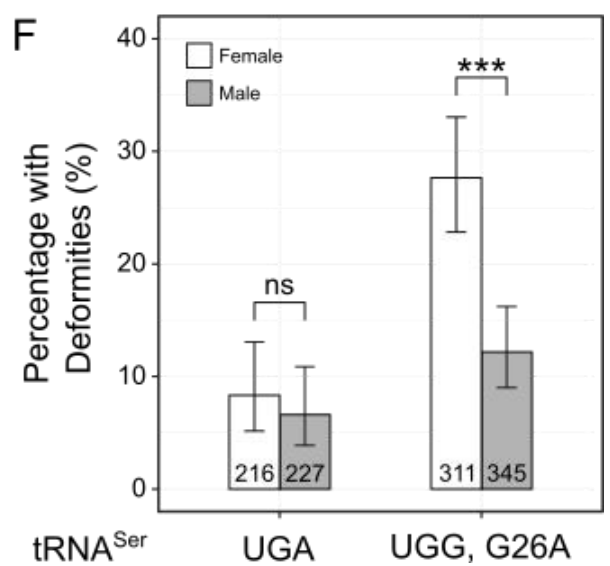

Figure 3. The $t R N A_{U G G, G 26 A}^{S e r}$ variant causes morphological deformities in adults in a sex-

specific manner. A) Examples of flies with misfused tergites, B) gnarled hindlegs, C) wing blisters, and D) missing wings/legs, as indicated by arrowheads. E) Percentage of tRNA ${ }_{\text {UGA }}^{\text {Ser }}$

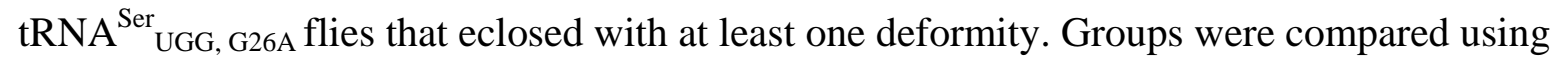
Fisher's exact test and corrected using the Holm-Bonferroni method. Bar height represents the percentage of flies of a genotype that had at least one deformity. Error bars represent the $95 \%$ confidence interval. Values within bars describe the number of flies examined for deformities. F) Same data as $\mathbf{E}$ but separated by sex. "ns" $\mathrm{p} \geq 0.05$, “*” $\mathrm{p}<0.05$, “**” $\mathrm{p}<0.01$, “***” $\mathrm{p}<0.001$. 
$t R N A_{U G G, G 26 A}^{\text {Ser }}$ impacts fly motility

Negative geotaxis assays are often used to study neurodegenerative diseases (e.g Feany

293 and Bender 2000; Song et al. 2017; Aggarwal et al. 2019), so as an initial examination of

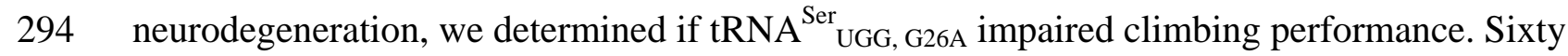

295 virgin, heterozygous flies of the four genotypes (tRNA ${ }_{\text {UGA }}^{\text {Ser }}$ males and females, and tRNA ${ }_{\text {UGG, }}^{\text {Ser }}$

296 G26A males and females) were collected and tested using a climbing assay every three days; flies

297 with deformities were not used in this experiment.

As expected, climbing performance of all genotypes decreased with age (F-tests

299 performed on generalized linear models corrected using Bonferroni’s method, Supplementary

300 File S2). For both males and females, climbing performance of tRNA ${ }_{\text {UGG, G26A flies was }}^{\text {Ser }}$

301 significantly worse than wild type tRNA ${ }_{\text {UGA }}^{\text {Ser }}$ flies (male: $p=0.001$, female: $p<0.001$, Figure

302 4A, B). Climbing performance was not significantly different when comparing males to females

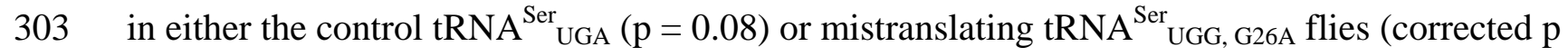

$304 \rightarrow 1$, Figure 4C, D). The climbing ability of male and female flies containing the wild type

305 tRNA ${ }_{\text {UGA }}^{\text {Ser }}$ declined at similar rates, as evidenced by the parallel performance curves $(p=0.97$,

306 Figure 4C). However, the climbing performance curve of female flies containing tRNA ${ }_{\text {UGG, }}^{\text {Ser }}$

307 G26A intersected the male curve, indicating that female climbing performance declined faster than

308 in males ( $\mathrm{p}=0.038$, Figure 4D, Supp. File S3). These data suggest that the mistranslating

309 tRNA $^{\text {Ser }}$ variant negatively affects locomotion and has an accelerated impact on female ability to

310 climb as they age. 

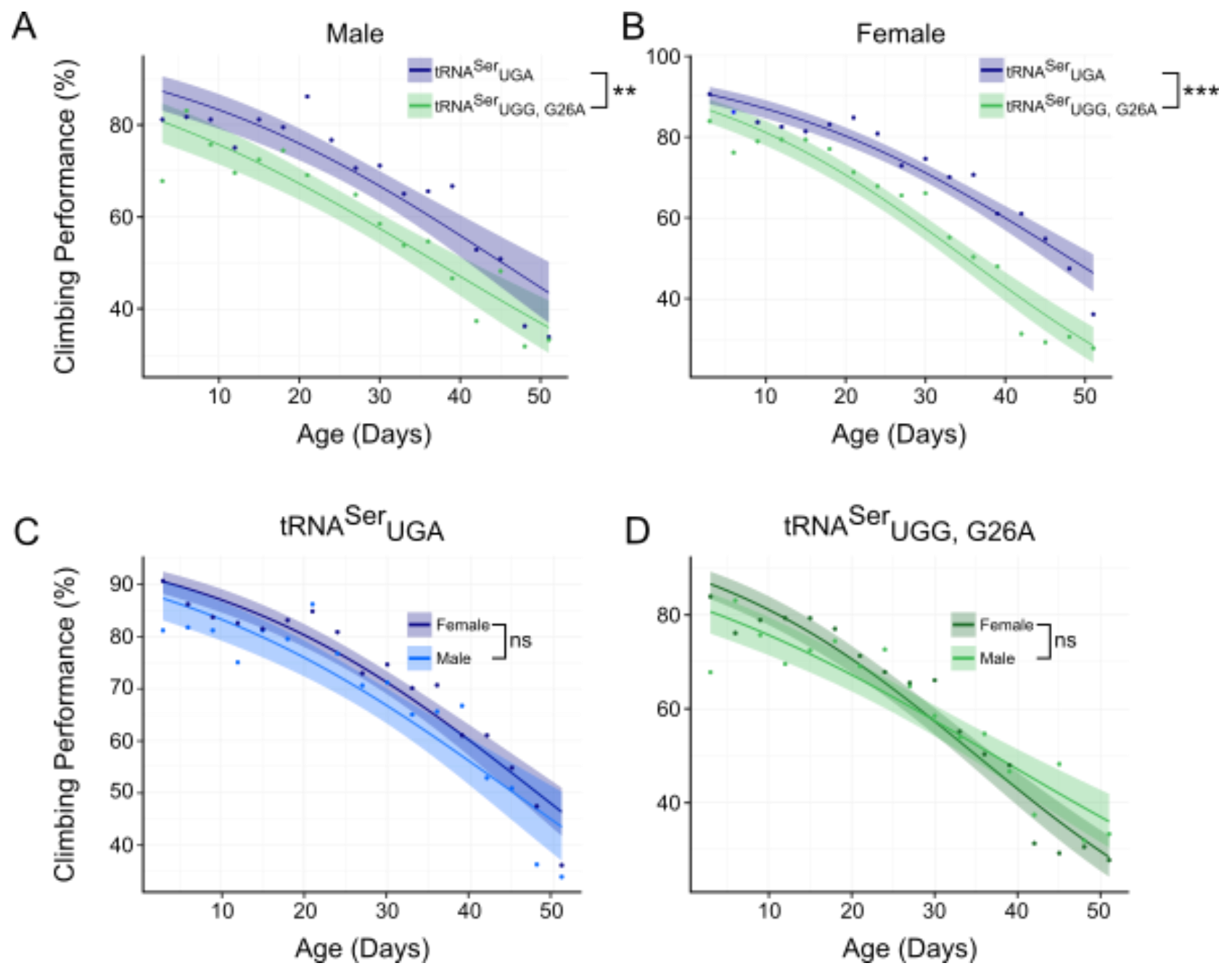

312 Figure 4. Fly locomotion is impacted by a mistranslating $t R N A^{\text {Ser }}$ variant. Each point represents

313 the percentage of flies (out of 60 from 11 vials) that managed to climb $5 \mathrm{~cm}$ in ten seconds

314 averaged over three trials. Generalized linear models were constructed from the performance

315 data and F-tests were performed on the models. P-values were corrected using Bonferroni's

316 method. Shaded region represents the $95 \%$ confidence intervals for the fitted performance

317 curves. A) Climbing performance of male flies containing tRNA ${ }_{\text {UGA }}^{\mathrm{Ser}}$ or tRNA ${ }_{\text {UGG, G26A. B) }}^{\text {Ser }}$

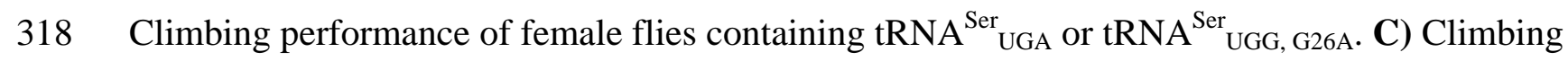

319 performance of male and female flies containing tRNA ${ }_{\text {UGA }}^{\text {Ser }}$ o) $t$ RNA ${ }_{\text {UGG, G26A. "ns" }}^{\text {Ser }} \geq$ 320 $0.05, “ * ” p<0.05, “ * * ” p<0.01, “ * * * ” p<0.001$. 


\section{DISCUSSION}

\section{Creating a fly model of mistranslation}

We have created a Drosophila melanogaster model containing a genomically-integrated

324 cytosolic tRNA that mistranslates serine for proline. The mistranslating fly model expands the

325 possibilities offered by yeast or cell lines by allowing for studies into sex-specific or tissue-

326 specific effects of mistranslating tRNA variants and the effect of tRNA variants on development

327 and disease.

Our method of transgene integration controlled for positional effects by inserting either

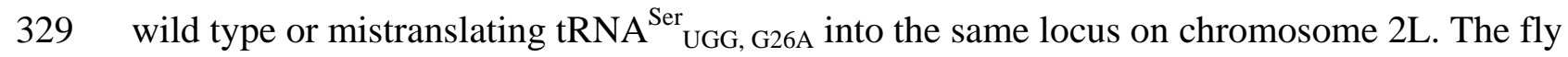

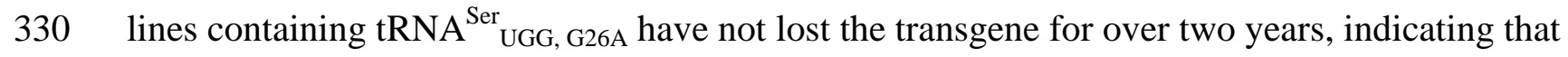

331 mistranslating tRNA variants can be stably maintained in the genome. We observed a proline-to-

332 serine misincorporation rate of $\sim 0.6 \%$ in the pupae for the genomically integrated tRNA ${ }_{\mathrm{UGG}}^{\mathrm{Ser}}$

333 G26A gene. This level of mistranslation was sufficient to cause deleterious phenotypes affecting

334 diverse aspects of fly physiology.

336 of the tRNA, stability and turnover of the tRNA variant, and number of competing tRNA genes.

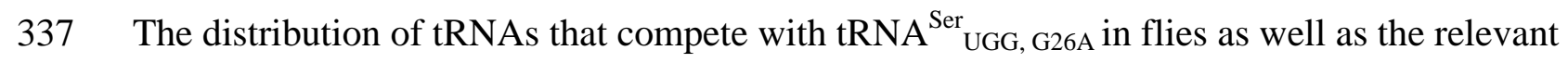

338 codon usage is shown in Supplemental File 1. Flies contain two proline tRNA species that

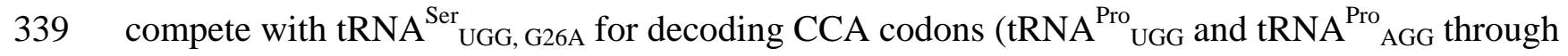

340 modification of A34 to inosine; Crick 1966; Boccaletto et al. 2018); likewise mistranslating

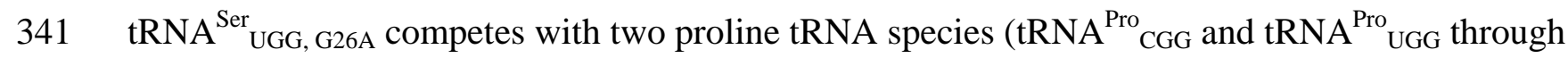

342 wobble pairing) for decoding CCG codons. The maximum frequency of mistranslation with a

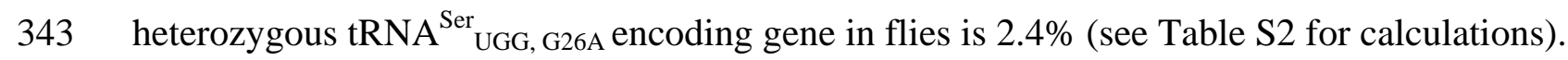


344 The less than maximal frequency of $0.6 \%$ observed for tRNA ${ }_{\text {UGG, G26A }}^{\text {Ser }}$ ispected because the

345 G26A mutation prevents dimethylation at position 26, which increases turnover by the rapid

346 tRNA decay pathway (Dewe et al. 2012).

347 A mistranslating $t R N A^{\text {Ser }}$ variant has diverse and sex-specific effects on flies

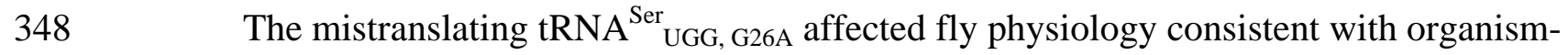

349 wide loss of proteostasis. Our findings resemble other studies of proteostasis loss in flies.

350 Impaired heat shock response exacerbates neurodegeneration and increases development time

351 (Warrick et al. 1999; Gong and Golic 2006), and many of the wing, leg and tergite deformities

352 observed for heterozygous Heat shock protein 83 (Hsp83) mutants look similar to those observed

353 in this study (Rutherford and Lindquist 1998). Developmental and neurodegenerative phenotypes

354 including locomotive defects as measured in a climbing assay were likewise observed in flies

355 containing a misacylation-prone PheRS (Lu et al. 2014). It is interesting to note that reduced

356 levels of translation lead to similar deformities as found in mistranslating flies. RNAi

357 knockdown of Nopp140, a gene involved in ribosome assembly, causes flies to present with

358 gnarled legs, missing wings, and misfused tergites (Cui and DiMario 2007). Minute genes

359 describe a collection of $>50$ genes required for protein synthesis. Their mutation results in

360 shorter, thinner bristles, delayed development, smaller body size, and anatomical deformities

361 when mutated (Schultz 1929; Marygold et al. 2007), again similar to the developmental and

362 anatomical aberrations seen in flies containing the mistranslating tRNA ${ }^{\text {Ser }}$ variant. Though

363 reduced translation and mistranslation are different processes, the similar phenotypes produced

364 demonstrate that development is highly dependent on accurate and efficient translation.

365 The increased impact of the mistranslating tRNA on female flies was striking. $D$.

366 melanogaster males and females have highly different physiology and experience different 
367 developmental challenges. Adult females are larger than males, develop faster, invest more

368 resources into reproduction, and tend to live longer than males (Bonnier 1926; Bakker 1959;

369 Sørensen et al. 2007; Ziehm et al. 2013). Males and females also display dimorphic responses to

370 proteotoxic stress. Fredriksson et al. (2012) examined protein carbonylation in female somatic

371 and germ line cells at different ages to determine how aging affects protein quality control of

372 somatic and reproductive tissues. They found that as females age, there are fewer carbonylated

373 proteins and reduced protein aggregation (both indicators of proteostasis loss) in eggs compared

374 to the soma. Their work shows that females prioritize proteostasis of their eggs over their

375 somatic cells, even while unmated. This trade-off could exacerbate the stress of mistranslating

376 tRNAs in females, particularly as they experience aging-induced loss of proteostasis, and could

377 contribute to the faster decline of climbing performance observed in female tRNA ${ }_{\text {UGG, G26A }}^{\text {Ser }}$

378 flies compared to males. Many stress-response pathways affect males and females differently.

379 For example, induction of the heat shock response increases male lifespan whereas female

380 lifespan is unaffected (Sørensen et al. 2007; reviewed in Tower 2011). Dietary restriction shows

381 the opposite trend, as it increases female lifespan more than male (Nakagawa et al. 2012; Regan

382 et al. 2016; reviewed in Garratt 2020). Experiments testing the effects of mistranslating tRNAs

383 on male and female fly longevity are ongoing. It is also possible that expression of the

384 mistranslating tRNA differs between males and females or that the mistranslating tRNA has

385 alternative functions (e.g. tRNA-derived fragments) that differ between males and females.

386 Implications for human disease

Our work suggests that mistranslating tRNA variants have the potential to influence

388 multiple aspects of human physiology. From a development perspective, the alteration in

389 progression through life stages and increased number of deformities suggest that the proteotoxic 
stress resulting from mistranslating tRNA variants may contribute to congenital or

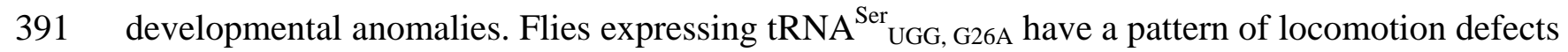

392 similar to those seen for flies expressing alleles associated with neurodegeneration (Feany and

393 Bender 2000; Song et al. 2017; Aggarwal et al. 2019). Interestingly, the mistranslating fly model

394 further resembles human neuropathies in that climbing performance declined faster in female

395 compared to male flies, just as some neurodegenerative disorders such as Alzheimer's and

396 Huntington's Disease, are more common or severe in women compared to men (Viña and Lloret

397 2010; Zielonka et al. 2013).

Given the prevalence of putative mistranslating tRNAs in the human population (Berg et mistranslating tRNAs can exacerbate diseases characterized by a loss of proteostasis (see also

401 Reverendo et al. 2014), and our results here indicate that these effects may differ in magnitude

402 between sexes. Our previous studies in yeast have shown negative genetic interactions between

403 mistranslation and mutations in genes involved in protein quality control and other pathways that

404 could contribute to disease (Hoffman et al. 2017; Berg et al. 2020, 2021). Our D. melanogaster

405 model of mistranslation allows for the expansion of these studies into the investigation of mutant

406 tRNA contribution to disease and development.

\section{Data availability}

408 Fly lines and plasmids are available upon request. The authors affirm that all data necessary for 409 confirming the conclusions of the article are present within the article, figures, and supplemental 410 material. Supplemental files are available at FigShare. Supplemental File S1 contains all 411 supplemental figures and tables. Supplemental File S2 contains all raw data. Supplemental File 
412 S3 contains R code used to analyze mass spectrometry, developmental, deformity, and climbing

413 assay data. Supplemental File S4 contains all images of deformed flies. The mass spectrometry

414 proteomics data have been deposited to the ProteomeXchange Consortium via the PRIDE partner

415 repository (Perez-Riverol et al. 2019) with the dataset identifier PXD028498.

\section{Acknowledgements}

417 We would like to thank the Biotron Integrated Microscopy facility for their aid with this

418 research. In particular, JRI would like to thank Karen Nygard, Reza Khazaee, and Marc

419 Courchesne for their instruction and help. We would also like to thank Dr. Yolanda Morbey for

420 guidance on analyzing the climbing assay data, Ricard Rodriguez-Mias for assisting with the

421 mass spectrometry and maintaining the instruments, and Julie Genereaux for technical

422 assistance.

\section{Funding}

424 This work was supported by NSERC grants to CJB [RGPIN-2015-04394] and AJM [RGPIN-

425 2020-06464], as well as a UWO MHSRB seed grant to AJM. Mass spectrometry work was

426 supported by a research grant from the Keck Foundation, NIH grant R35 GM119536 and

427 associated instrumentation supplement to JV. JRI and MDB were supported by an NSERC PGS-

428 D and NSERC CGS-D respectively.

\section{Conflicts of Interest}

430 The authors declare that there was no conflict of interest while conducting and reporting this

431 research. 


\section{Literature Cited}

434 Achsel T., and H. J. Gross, 1993 Identity determinants of human tRNA ${ }^{\text {Ser. }}$ : sequence elements

435 necessary for serylation and maturation of a tRNA with a long extra arm. EMBO J. 12:

$436 \quad 3333-3338$.

437

438

439

Aggarwal A., H. Reichert, and K. VijayRaghavan, 2019 A locomotor assay reveals deficits in heterozygous Parkinson's disease model and proprioceptive mutants in adult Drosophila. Proc. Natl. Acad. Sci. USA. 116: 24830-24839.

Asahara H., H. Himeno, K. Tamura, T. Hasegawa, K. Watanabe, et al., 1993 Recognition nucleotides of Escherichia coli $\mathrm{tRNA}^{\mathrm{Leu}}$ and its elements facilitating discrimination from tRNA $^{\text {Ser }}$ and tRNA ${ }^{\text {Tyr }}$. J. Mol. Biol. 231: 219-229.

Bakker K., 1959 Feeding period, growth, and pupation in larvae of Drosophila melanogaster. Entomol. Exp. Appl. 2: 171-186.

Berg M. D., K. S. Hoffman, J. Genereaux, S. Mian, R. S. Trussler, et al., 2017 Evolving mistranslating tRNAs through a phenotypically ambivalent intermediate in Saccharomyces cerevisiae. Genetics 206: 1865-1879.

Berg M. D., D. J. Giguere, J. S. Dron, J. T. Lant, J. Genereaux, et al., 2019a Targeted sequencing reveals expanded genetic diversity of human transfer RNAs. RNA Biol. 16: 1574-1585.

Berg M. D., Y. Zhu, J. Genereaux, B. Y. Ruiz, R. A. Rodriguez-Mias, et al., 2019b Modulating mistranslation potential of tRNA ${ }^{\mathrm{Ser}}$ in Saccharomyces cerevisiae. Genetics 213: 849-863.

Berg M. D., Y. Zhu, J. Isaacson, J. Genereaux, R. Loll-Krippleber, et al., 2020 Chemical-genetic interactions with the proline analog 1-azetidine-2-carboxylic acid in Saccharomyces 
cerevisiae. G3 Genes|Genomes|Genetics 10: 4335-4345.

455

456

457

Berg M. D., Y. Zhu, B. Y. Ruiz, R. Loll-Krippleber, J. Isaacson, et al., 2021 The amino acid substitution affects cellular response to mistranslation. G3 Genes|Genomes|Genetics jkab218.

Boccaletto P., M. A. MacHnicka, E. Purta, P. Pitkowski, B. Baginski, et al., 2018 MODOMICS: a database of RNA modification pathways. 2017 update. Nucleic Acids Res. 46: D303D307.

Bonnier G., 1926 Temperature and time of development of the two sexes in Drosophila. J. Exp. Biol. 4: 186-195.

Breitschopf K., T. Achsel, K. Busch, and H. J. Gross, 1995 Identity elements of human tRNA ${ }^{\text {Leu. }}$ structural requirements for converting human tRNA ${ }^{\text {Ser }}$ into a leucine acceptor in vitro. Nucleic Acids Res. 23: 3633-3637.

Chan P. P., and T. M. Lowe, 2016 GtRNAdb 2.0: An expanded database of transfer RNA genes identified in complete and draft genomes. Nucleic Acids Res. 44: D184-D189.

Chiu Y. H., and N. R. Morris, 1997 Genetic and molecular analysis of a tRNA ${ }^{\text {Leu }}$ missense suppressor of nudC3, a mutation that blocks nuclear migration in Aspergillus nidulans. Genetics 145: 707-714.

Crick F. H. C., 1966 Codon—anticodon pairing: the wobble hypothesis. J. Mol. Biol. 19: 548555.

Cui Z., and P. J. DiMario, 2007 RNAi knockdown of Nopp140 induces Minute-like phenotypes in Drosophila. Mol. Biol. Cell 18: 2179-2191. 
Dewe J. M., J. M. Whipple, I. Chernyakov, L. N. Jaramillo, and E. M. Phizicky, 2012 The yeast rapid tRNA decay pathway competes with elongation factor 1A for substrate tRNAs and acts on tRNAs lacking one or more of several modifications. RNA 18: 1886-1896.

Eng J. K., T. A. Jahan, and M. R. Hoopmann, 2013 Comet: An open-source MS/MS sequence database search tool. Proteomics 13: 22-24.

Feany M. B., and W. W. Bender, 2000 A Drosophila model of Parkinson's disease. Nature 404: 394-398. of aging and reproduction on protein quality control in soma and gametes of Drosophila

Garratt M., 2020 Why do sexes differ in lifespan extension? Sex-specific pathways of aging and melanogaster. Aging Cell 11: 634-643.

Garza D., M. M. Medhora, and D. L. Hartl, 1990 Drosophila nonsense suppressors: functional underlying mechanisms for dimorphic responses. Nutr. Heal. Aging 5: 247-259. tRNAs as tools to induce proteome damage and identify components of stress responses. Nucleic Acids Res. 38: e30.

493 Giegé R., M. Sissler, and C. Florentz, 1998 Universal rules and idiosyncratic features in tRNA identity. Nucleic Acids Res. 26: 5017-5035. 
vitro mutagenized yeast genes lacking six-base pair restriction sites. Gene 74: 527-534.

497 Gong W. J., and K. G. Golic, 2006 Loss of Hsp70 in Drosophila is pleiotropic, with effects on

498 thermotolerance, recovery from heat shock and neurodegeneration. Genetics 172: 275-286.

499 Goodman H. M., J. Abelson, A. Landy, S. Brenner, and J. D. Smith, 1968 Amber suppression: a

$500 \quad$ nucleotide change in the anticodon of a tyrosine transfer RNA. Nature 217: 1019-1024.

501 Goodman H. M., M. V. Olson, and B. D. Hall, 1977 Nucleotide sequence of a mutant eukaryotic

502 gene: the yeast tyrosine-inserting ochre suppressor SUP4-o. Proc. Natl. Acad. Sci. USA. 74:

$503 \quad 5453-5457$.

504 Gorini L., and J. R. Beckwith, 1966 Suppression. Annu. Rev. Microbiol. 20: 401-422.

505 Goto Y. I., I. Nonaka, and S. Horai, 1990 A mutation in the tRNA ${ }^{\text {Leu(UUR) }}$ gene associated with

506 the MELAS subgroup of mitochondrial encephalomyopathies. Nature 348: 651-653.

507 Himeno H., S. Yoshida, A. Soma, and K. Nishikawa, 1997 Only one nucleotide insertion to the

$508 \quad$ long variable arm confers an efficient serine acceptor activity upon Saccharomyces

$509 \quad$ cerevisiae tRNA $^{\text {Leu }}$ in vitro. J. Mol. Biol. 268: 704-711.

510 Hoffman K. S., M. D. Berg, B. H. Shilton, C. J. Brandl, and P. O’Donoghue, 2017 Genetic

511 selection for mistranslation rescues a defective co-chaperone in yeast. Nucleic Acids Res.

$512 \quad 45: 3407-3421$.

513 Hou Y. M., and P. Schimmel, 1988 A simple structural feature is a major determinant of the

514 identity of a transfer RNA. Nature 333: 140-145.

515 Isaacson J., 2018 Creating tools to determine whether Katanin 60 affects female rejection of

516 males in Drosophila. Electron. Thesis Diss. Repos. 5588: https://ir.lib.uwo.ca/etd/5588. 
Ishimura R., G. Nagy, I. Dotu, H. Zhou, X. L. Yang, et al., 2014 Ribosome stalling induced by mutation of a CNS-specific tRNA causes neurodegeneration. Science. 345: 455-459.

Joshi K., L. Cao, and P. J. Farabaugh, 2019 The problem of genetic code misreading during protein synthesis. Yeast 36: 35-42.

Käll L., J. D. Canterbury, J. Weston, W. S. Noble, and M. J. MacCoss, 2007 Semi-supervised learning for peptide identification from shotgun proteomics datasets. Nat. Methods 4: 923-

Kapur M., and S. L. Ackerman, 2018 mRNA translation gone awry: translation fidelity and

Lant J. T., M. D. Berg, I. U. Heinemann, C. J. Brandl, and P. O’Donoghue, 2019 Pathways to 925.

Laski F. A., S. Ganguly, P. A. Sharp, U. L. RajBhandary, and G. M. Rubin, 1989 Construction, stable transformation, and function of an amber suppressor tRNA gene in Drosophila

Leutert M., R. A. Rodríguez-Mias, N. K. Fukuda, and J. Villén, 2019 R2-P2 rapid-robotic phosphoproteomics enables multidimensional cell signaling studies. Mol. Syst. Biol. 15: melanogaster. Proc. Natl. Acad. Sci. USA. 86: 6696-6698. 
Lu J., M. Bergert, A. Walther, and B. Suter, 2014 Double-sieving-defective aminoacyl-tRNA synthetase causes protein mistranslation and affects cellular physiology and development. Nat. Commun. 5: 1-13.

Marygold S. J., J. Roote, G. Reuter, A. Lambertsson, M. Ashburner, et al., 2007 The ribosomal protein genes and Minute loci of Drosophila melanogaster. Genome Biol. 8.

543 Mcclain W. H., and K. Foss, 1988 Changing the identity of a tRNA by introducing a G-U wobble pair near the 3' acceptor end. Science. 240: 793-796.

Meziane A. El, S. K. Lehtinen, N. Hance, L. G. J. Nijtmans, D. Dunbar, et al., 1998 A tRNA suppressor mutation in human mitochondria. Nat. Genet. 18: 350-353.

Mitchell H. K., L. S. Lipps, and U. M. Tracy, 1977 Transcriptional changes in pupal hypoderm

Mitchell H. K., and N. S. Petersen, 1981 Rapid changes in gene expression in differentiating tissues of Drosophila. Dev. Biol. 85: 233-242.

Murakami S., K. Kuehnle, and D. B. Stern, 2005 A spontaneous tRNA suppressor of a mutation in the Chlamydomonas reinhardtii nuclear MCD1 gene required for stability of the chloroplast petD mRNA. Nucleic Acids Res. 33: 3372-3380.

554 Nakagawa S., M. Lagisz, K. L. Hector, and H. G. Spencer, 2012 Comparative and meta-analytic insights into life extension via dietary restriction. Aging Cell 11: 401-409.

Nangle L. A., C. M. Motta, and P. Schimmel, 2006 Global effects of mistranslation from an editing defect in mammalian cells. Chem. Biol. 13: 1091-1100. 
and chemically triggered oxidative stress modifies translational fidelity. Nature 462: 522526.

Normanly J., T. Ollick, and J. Abelson, 1992 Eight base changes are sufficient to convert a leucine-inserting tRNA into a serine-inserting tRNA. Proc. Natl. Acad. Sci. USA. 89: 56805684.

Paredes J. A., L. Carreto, J. Simões, A. R. Bezerra, A. C. Gomes, et al., 2012 Low level genome mistranslations deregulate the transcriptome and translatome and generate proteotoxic stress

Pina C., and F. Pignoni, 2012 Tubby-RFP balancers for developmental analysis: $F M 7 c 2 x T b$ in yeast. BMC Biol. 10: 55.

Port F., H. M. Chen, T. Lee, and S. L. Bullock, 2014 Optimized CRISPR/Cas tools for efficient germline and somatic genome engineering in Drosophila. Proc. Natl. Acad. Sci. USA. 111:

Regan J. C., M. Khericha, A. J. Dobson, E. Bolukbasi, N. Rattanavirotkul, et al., 2016 Sex difference in pathology of the ageing gut mediates the greater response of female lifespan to dietary restriction. eLife 5: e10956.

Reverendo M., A. R. Soares, P. M. Pereira, L. Carreto, V. Ferreira, et al., 2014 tRNA mutations that affect decoding fidelity deregulate development and the proteostasis network in zebrafish. RNA Biol. 11: 1199-1213. mistranslation caused by an ambiguous genetic code. Proc. Natl. Acad. Sci. USA. 105: 
$16502-16507$.

581 Rutherford S. L., and S. Lindquist, 1998 Hsp90 as a capacitor for morphological evolution.

$582 \quad$ Nature 396: 336-342.

583 Samhita L., P. K. Raval, and D. Agashe, 2020 Global mistranslation increases cell survival under $584 \quad$ stress in Escherichia coli. PLoS Genet. 16: e1008654.

585 Schoenmakers E., B. Carlson, M. Agostini, C. Moran, O. Rajanayagam, et al., 2016 Mutation in

586 human selenocysteine transfer RNA selectively disrupts selenoprotein synthesis. J. Clin.

$587 \quad$ Invest. 126: 992-996.

588 Schultz J., 1929 The Minute reaction in the development of Drosophila melanogaster. Genetics 14: $366-419$.

590 Shan G., and S. Gerstenberger, 2017 Fisher's exact approach for post hoc analysis of a chisquared test. PLoS One 12: e0188709. mistranslation leads to silencing of the unfolded protein response and increased

Shoffner J. M., M. T. Lott, A. M. S. Lezza, P. Seibel, S. W. Ballinger, et al., 1990 Myoclonic epilepsy and ragged-red fiber disease (MERRF) is associated with a mitochondrial DNA mitochondrial biogenesis. Commun. Biol. 2: 381.

Sikorski R. S., and P. Hieter, 1989 A system of shuttle vectors and yeast host strains designed for efficient manipulation of DNA in Saccharomyces cerevisiae. Genetics 122: 19-27. 
deficits and dopaminergic neuron loss in a Drosophila model of Parkinson's disease. Cell Rep. 18: 1132-1143.

Sørensen J. G., T. N. Kristensen, K. V. Kristensen, and V. Loeschcke, 2007 Sex specific effects of heat induced hormesis in Hsf-deficient Drosophila melanogaster. Exp. Gerontol. 42: $1123-1129$.

Stadler J., and C. Yanofsky, 1959 Studies on a series of tryptophan-independent strains derived from a tryptophan-requiring mutant of Escherichia coli. Genetics 44: 105-123.

Tower J., 2011 Heat shock proteins and Drosophila aging. Exp. Gerontol. 46: 355-362.

Viña J., and A. Lloret, 2010 Why women have more Alzheimer's disease than men: Gender and mitochondrial toxicity of amyloid- $\beta$ peptide. J. Alzheimer's Dis. 20.

611 Warrick J. M., H. Y. E. Chan, G. L. Gray-Board, Y. Chai, H. L. Paulson, et al., 1999 Suppression of polyglutamine-mediated neurodegeneration in Drosophila by the molecular

614 Wills N., R. F. Gesteland, J. Karn, L. Barnett, S. Bolten, et al., 1983 The genes sup-7 X and sup5 III of C. elegans suppress amber nonsense mutations via altered transfer RNA. Cell 33:

617 Wiltrout E., J. M. Goodenbour, M. Fréchin, and T. Pan, 2012 Misacylation of tRNA with methionine in Saccharomyces cerevisiae. Nucleic Acids Res. 40: 10494-10506.

619 Zheng J., Y. Ji, and M. X. Guan, 2012 Mitochondrial tRNA mutations associated with deafness.

$620 \quad$ Mitochondrion 12: 406-413.

621 Ziehm M., M. D. Piper, and J. M. Thornton, 2013 Analysing variation in Drosophila aging 
across independent experimental studies: a meta-analysis of survival data. Aging Cell 12:

623 $917-922$.

624 Zielonka D., J. Marinus, R. A. C. Roos, G. De Michele, S. Di Donato, et al., 2013 The influence 625 of gender on phenotype and disease progression in patients with Huntington's disease. Park.

626 Relat. Disord. 19: 192-197.

627 Zimmerman S. M., Y. Kon, A. C. Hauke, B. Y. Ruiz, S. Fields, et al., 2018 Conditional

628 accumulation of toxic tRNAs to cause amino acid misincorporation. Nucleic Acids Res. 46:

629 7831-7843. 